\title{
Physical Activity of Pregnant Hispanic Women
}

\author{
Kristine E. Lynch, PhD, Jill R. Landsbaugh, PhD, Brian W. Whitcomb, PhD, Penny Pekow, \\ PhD, Glenn Markenson, MD, and Lisa Chasan-Taber, ScD \\ Division of Biostatistics and Epidemiology (Lynch, Whitcomb, Pekow, Chasan-Taber), School of \\ Public Health and Health Sciences, University of Massachusetts at Amherst, Amherst; Baystate \\ Medical Center (Markenson), Springfield, Massachusetts; Medical Research Council \\ Epidemiology Unit (Landsbaugh), Cambridge, United Kingdom
}

\begin{abstract}
Background-A growing body of evidence suggests that physical activity during pregnancy can reduce risk of pregnancy complications. However, factors influencing activity in pregnant Hispanic women, who have high rates of sedentary activity as compared to non-Hispanic whites, are not well characterized.
\end{abstract}

Purpose-To assess patterns and correlates of physical activity among 1355 participants in Proyecto Buena Salud, a prospective cohort of pregnant Hispanic women in Massachusetts from 2006 to 2011.

Methods-Analyses were conducted in 2012. Pre-, early-, mid-, and late-pregnancy physical activity were assessed using the Pregnancy Physical Activity Questionnaire. Women reported the frequency and duration of household/caregiving, occupational, sports/exercise, and transportation activities, and were classified according to compliance with American College of Obstetricians and Gynecologists guidelines for physical activity.

Results-Household/caregiving activity was the primary mode of pregnancy activity ranging from $56 \%$ to $60 \%$ of total activity while sports/exercise contributed the least $(<10 \%)$. Compared to nulliparous women, women with two or more children were $85 \%$ less likely to become inactive at any time during pregnancy $(\mathrm{OR}=0.15[95 \% \mathrm{CI}=0.04,0.56], p$-trend $<0.01)$. Women with one or more children increased their total physical activity on average 9.73 \pm 2.04 MET hours/week and 12.04 \pm 2.39 MET-hours/week, respectively, with the onset of pregnancy $(p<0.01)$. Those with the highest levels of total physical activity prior to pregnancy were $87 \%$ less likely to become inactive with the onset of pregnancy than those who were inactive prior to pregnancy $(\mathrm{OR}=0.13[95 \% \mathrm{CI}=$ $0.05,0.29])$.

Conclusions-Findings can inform culturally appropriate interventions designed to reduce pregnancy complications through the promotion of physical activity during pregnancy.

(C) 2012 American Journal of Preventive Medicine. Published by Elsevier Inc. All rights reserved.

Address correspondence to: Kristine E. Lynch, PhD, 420 Arnold House, 715 N Pleasant St., Amherst MA 01003.

Kelyn0@schoolph.umass.edu.

No financial disclosures were reported by the authors of this paper.

Publisher's Disclaimer: This is a PDF file of an unedited manuscript that has been accepted for publication. As a service to our customers we are providing this early version of the manuscript. The manuscript will undergo copyediting, typesetting, and review of the resulting proof before it is published in its final citable form. Please note that during the production process errors may be discovered which could affect the content, and all legal disclaimers that apply to the journal pertain. 


\section{Introduction}

Physical activity during pregnancy has been associated with reduced risk of pregnancy complications such as excessive gestational weight gain, Caesarean delivery, ${ }^{1}$ preeclampsia, and gestational diabetes mellitus (GDM) ${ }^{2-4}$ However, physical activity declines with the onset of pregnancy ${ }^{5-7}$ and the majority of women fail to meet the American College of Obstetricians and Gynecologists (ACOG) physical activity guidelines for pregnancy. ${ }^{8}$

Hispanics are half as likely as non-Hispanic whites to meet ACOG guidelines. ${ }^{9}$ This is critical as Hispanics are the largest minority group in the U.S. with the highest birth and immigration rates. ${ }^{10}$ The majority of prior studies that examined pregnancy physical activity were conducted in predominantly non-Hispanic populations and were often limited by assessment tools not validated in pregnant women or focused exclusively on sports/ exercise. ${ }^{7,11-20}$

The present study examined patterns and predictors of change in physical activity levels throughout pregnancy in a prospective study of pregnant Hispanic women. Prior studies have suggested that sociodemographics, behavioral, and psychosocial factors influence physical activity beliefs and behaviors. Therefore, it was hypothesized that physical activity levels would decrease throughout pregnancy, and that sociodemographic, behavioral, and psychosocial factors would predict change in physical activity during pregnancy.

\section{Methods}

\section{Study Design and Participants}

Proyecto Buena Salud (PBS) was a prospective cohort study of prenatal care patients of Puerto Rican/Dominican heritage conducted at Baystate Medical Center in Springfield, Massachusetts from 2006 to $2011 .^{21}$ This study was approved by the IRBs of the University of Massachusetts-Amherst and Baystate Health. All participants provided written informed consent. Women were recruited from the ambulatory obstetric practices if they were at 20 weeks' gestation. Exclusion criteria included nonsingleton pregnancy, history of diabetes, hypertension, heart disease or chronic renal disease, and being aged $<16$ years or $>40$ years.

\section{Variable Assessment}

Bilingual study personnel interviewed participants in early-, mid-, and late pregnancy. Physical activity was measured via the Pregnancy Physical Activity Questionnaire (PPAQ). ${ }^{22}$ The PPAQ is a semiquantitative questionnaire that asks respondents to report time spent participating in 32 activities including household/caregiving (13 activities); occupational (five activities); sports/exercise (eight activities); transportation (three activities); and inactivity (three activities). Women were categorized according to whether they achieved ACOG guidelines ( $>30$ minutes/day physical activity of at least moderate intensity on most days of the week). Acculturation was assessed using the Psychological Acculturation Scale (PAS). ${ }^{23}$ Depressive symptoms were assessed using the ten-item Edinburgh Postnatal Depression Scale (EPDS). ${ }^{24}$ Perceived stress was measured using Cohen's Perceived Stress Scale (PSS-14), ${ }^{25}$ and trait anxiety was assessed using the Spielberger State-Trait Anxiety Inventory (STAI). ${ }^{26}$

\section{Statistical Analysis}

All analyses were conducted with SAS 9.3 in 2012. Logistic regression was used to assess predictors of becoming inactive (not meeting ACOG guidelines) during each pregnancy time period, and overall, restricting analyses to women who met ACOG guidelines in the prior 
period. Linear regression was used to assess predictors of change in MET-hours/week of each type and intensity from pre- to early pregnancy. Initial models included factors observed to be associated with change in physical activity in the prior literature (e.g., age, BMI, education, income, and parity) as well as factors that were significant in univariate models at $p<0.20$. Final models retained those variables observed to change beta coefficients for physical activity by at least $10 \%$.

\section{Results}

The mean age of participants was $23.7 \pm 5.7$ years. The majority were unmarried (89\%) and had one or more live births (59\%); approximately half were overweight or obese prior to pregnancy (45\%). One quarter (24.7\%) met ACOG guidelines for sports/exercise activity in pre-pregnancy and $7.1 \%$ met ACOG guidelines in early pregnancy. When household/ caregiving, transportation, and occupational activities were also taken into account, a total of $69.7 \%$ of women met ACOG guidelines in pre-pregnancy and $45 \%$ in early pregnancy.

Household/caregiving activities represented the largest component of pre-pregnancy activity (49.9\%) and throughout pregnancy (ranging from 56\% to 60\%), while sports/exercise represented the smallest proportion of pregnancy activity (ranging from $7.6 \%$ to $8.9 \%$ ). Significant within-woman decreases for all activity-intensity and activity-domain categories occurred from pre-pregnancy to early pregnancy $(p<0.01)$ with the largest decrease of 11.6 MET-hours/week observed for moderate-intensity activities.

Compared to nulliparous women, women with one or more children were less likely to become inactive at any time during pregnancy $(\mathrm{OR}=0.15$ [95\% $\mathrm{CI}=0.04,0.56], p$-trend $<0.01$; Table 1). Compared to normal-weight women, obese women who had been active prior to pregnancy were less likely to become inactive at any time during pregnancy $(\mathrm{OR}=0.29[95 \% \mathrm{CI}=0.10,0.82])$. Women with the highest levels of pre-pregnancy activity were less likely to become inactive with pregnancy onset versus women with the lowest levels $(\mathrm{OR}=0.13$ [95\% CI=0.05, 0.29], $p$-trend <0.01). Similar findings were observed during the transition from pre- to mid-pregnancy, and pre-pregnancy to late pregnancy.

Women with children increased their total physical activity on average 9-12 MET hours/ week ( $p<0.01)$ with pregnancy onset as compared to nulliparous women, largely due to an increase in moderate-intensity activity ( $\beta=8.23$ MET-hours/week; $p<0.01$; Table 2$)$. Women aged $16-19$ years $(\beta=-1.78$ MET-hours/week; $p<0.01)$ and women who completed college $(\beta=-1.52$ MET-hours/week; $p<0.01)$ decreased their sedentary activity compared to those aged 20-24 years and to those with less than a high school education, respectively. Women with two or more children increased their occupational activity by 4.26 MET-hours/week and sports/exercise by 3.43 MET-hours/week compared to women with no children $(p<0.01)$. Women who were the most active in pre-pregnancy decreased their total activity 43.57 MET-hours/week more than the least-active women $(p<0.01)$.

\section{Conclusion}

In this prospective study among Hispanic women, few women met ACOG guidelines for sports/exercise activity in pregnancy; however, rates increased when considering activity of any type. Parous women and those with the highest levels of pre-pregnancy activity were less likely to become inactive at any point during pregnancy.

Findings that women with children were less likely to become inactive throughout pregnancy are in agreement with some prior studies. ${ }^{6,20,27}$ In contrast, studies of primarily non-Hispanic white women found parity to be positively associated with becoming insufficiently active during pregnancy; ${ }^{11,15,19,28,29}$ however, these studies assessed only 
sports/exercise. Household/caregiving activities make up the majority of total pregnancy activity among Hispanics. ${ }^{6,27}$ The fact that the PPAQ incorporates household/caregiving activities as part of the measure of total physical activity may explain, in part, these differences in findings.

Women with higher BMI who were active prior to pregnancy were less likely to become inactive during pregnancy. These results are consistent with prior literature ${ }^{13,27}$ and may be due to true differences or to over-reporting of activities such as walking by active overweight women. Zhang et al. found that women with a higher ponderal index prior to pregnancy reported higher levels of walking and calisthenics as compared to leaner women. ${ }^{29}$ The self-reported measure of physical activity is subject to potential misclassification that would tend to bias the results toward the null. However, this concern was minimized by the use of a PPAQ developed and validated in the study population and administered by bilingual interviewers.

Results from this study identify predictors of change in physical activity in a high-risk Puerto Rican/Dominican heritage population. Findings suggest that measurement of household/caregiving activities and other obligatory activities (e.g., occupational and transportation) in future studies may help to avoid misclassifying women as sedentary when the opposite might be true. Finally, the significant positive relationship identified between pre-pregnancy and pregnancy exercise suggests that future interventions should target inactive women prior to the onset of pregnancy. Such programs should involve counseling women by providing them with information on prenatal physical activity guidelines and encouraging them to adopt lifestyle changes to minimize the risk of pregnancy complications. Providers should also help their prenatal care patients identify strategies to overcome barriers to adopting and maintaining prenatal physical activity.

\section{Acknowledgments}

This work was supported by NIH NIDDK064902.

\section{References}

1. Bungum TJ. Exercise during pregnancy and type of delivery in nulliparae. J Obstet Gynecol Neonatal Nurs. 2000; 29(3):258-264.

2. Oken E. Associations of physical activity and inactivity before and during pregnancy with glucose tolerance. Obstet Gynecol. 2006; 108(5):1200-1007. [PubMed: 17077243]

3. Chasan-Taber L. Physical activity and gestational diabetes mellitus among Hispanic women. J Womens Health. 2008; 17(6):999-1008.

4. Zhang C. A prospective study of pregravid physical activity and sedentary behaviors in relation to the risk for gestational diabetes mellitus. Arch Intern Med. 2006; 166(5):543-548. [PubMed: 16534041]

5. Poudevigne MS. A review of physical activity patterns in pregnant women and their relationship to psychological health. Sports Med. 2006; 36(1):19-38. [PubMed: 16445309]

6. Schmidt MD. Physical activity patterns during pregnancy in a diverse population of women. $\mathrm{J}$ Womens Health. 2006; 15(8):909-918.

7. Borodulin KM. Physical activity patterns during pregnancy. Med Sci Sports Exerc. 2008; 40(11): 1901-1908. [PubMed: 18845974]

8. ACOG Committee Obstetric Practice. ACOG committee opinion: Number 267, January 2002: Exercise during pregnancy and the postpartum period. Obstet Gynecol. 2002; 99:171-173. [PubMed: 11777528]

9. Evenson KR. National trends in self-reported physical activity and sedentary behaviors among pregnant women: NHANES 1999-2006. Prev Med. 2010; 50(3):123-128. [PubMed: 20053370] 
10. Zambrana RE. Health data issues for Hispanics: Implications for public health research. J Health Care Poor Underserved. 2010; 12(1):20-34. [PubMed: 11217225]

11. Jukic MZ. Correlates of physical activity at two time points during pregnancy. J Phys Act Health. 2012; 9(3):325-335. [PubMed: 22454434]

12. Orr S, James S, Garry J, Prince C, Newton E. Exercise and pregnancy outcome among urban, lowincome, black women. Ethn Dis. 2006; 16(4):933-937. [PubMed: 17061749]

13. Hinton PS. Predictors of pregnancy-associated change in physical activity in a rural white population. Matern Child Health J. 2001; 5(1):7-14. [PubMed: 11341722]

14. Evenson KR. Leisure time physical activity among pregnant women in the U. S Paediatr Perinat Epidemiol. 2004; 18(6):400-407.

15. Pereira MA. Predictors of change in physical activity during and after pregnancy: Project Viva. Am J Prev Med. 2007; 32(4):312-319. [PubMed: 17383562]

16. Petersen AM. Correlates of physical activity among pregnant women in the U. S Med Sci Sports Exerc. 2005; 37(10):1748-1753.

17. Hegaard HK. Leisure time physical activity is associated with a reduced risk of preterm delivery. Am J Obstet Gynecol. 2008; 198(2):180.e1-180.e5. [PubMed: 18226619]

18. Borodulin K. Physical activity patterns during pregnancy through postpartum. BMC Womens Health. 2009; 9(1):32-38. [PubMed: 19925650]

19. Mottola MF. Activity patterns during pregnancy. Can J Appl Physiol. 2003; 28(4):642-653. [PubMed: 12904639]

20. Liu J. Physical activity during pregnancy in a prospective cohort of British women: Results from the Avon longitudinal study of parents and children. Eur J Epidemiol. 2011; 26(3):237-247. [PubMed: 21191632]

21. Chasan-Taber L. A prospective cohort study of modifiable risk factors for gestational diabetes among Hispanic women: Design and baseline characteristics. J Womens Health. 2010; 19(1):117124.

22. Chasan-Taber L. Development and validation of a pregnancy physical activity questionnaire. Med Sci Sports Exerc. 2004; 36(10):1750-1760. [PubMed: 15595297]

23. Troop LR. Psychological acculturation: Development of a new measure for Puerto Ricans on the U.S. mainland. Educ Psychol Meas. 1999; 59(2):351-367. [PubMed: 21415932]

24. Jadresic E. Validation of the Edinburgh postnatal depression scale (EPDS) in Chilean postpartum women. J Psychosom Obstet Gynaecol. 1995; 16(4):187-191. [PubMed: 8748993]

25. Cohen S. A global measure of perceived stress. J Health Soc Behav. 1983:385-396. [PubMed: 6668417]

26. Spielberger CD. State-Trait anxiety inventory. Corsini Encyclopedia of Psychology. 1996

27. Chasan-Taber L. Correlates of physical activity in pregnancy among Latina women. Matern Child Health J. 2007; 11(4):353-563. [PubMed: 17345155]

28. Ning Y. Correlates of recreational physical activity in early pregnancy. J Matern Fetal Neonatal Med. 2003; 13(6):385-393. [PubMed: 12962263]

29. Zhang J. Exercise during pregnancy among U.S. women. Ann Epidemiol. 1996; 6(1):53-59. [PubMed: 8680626] 


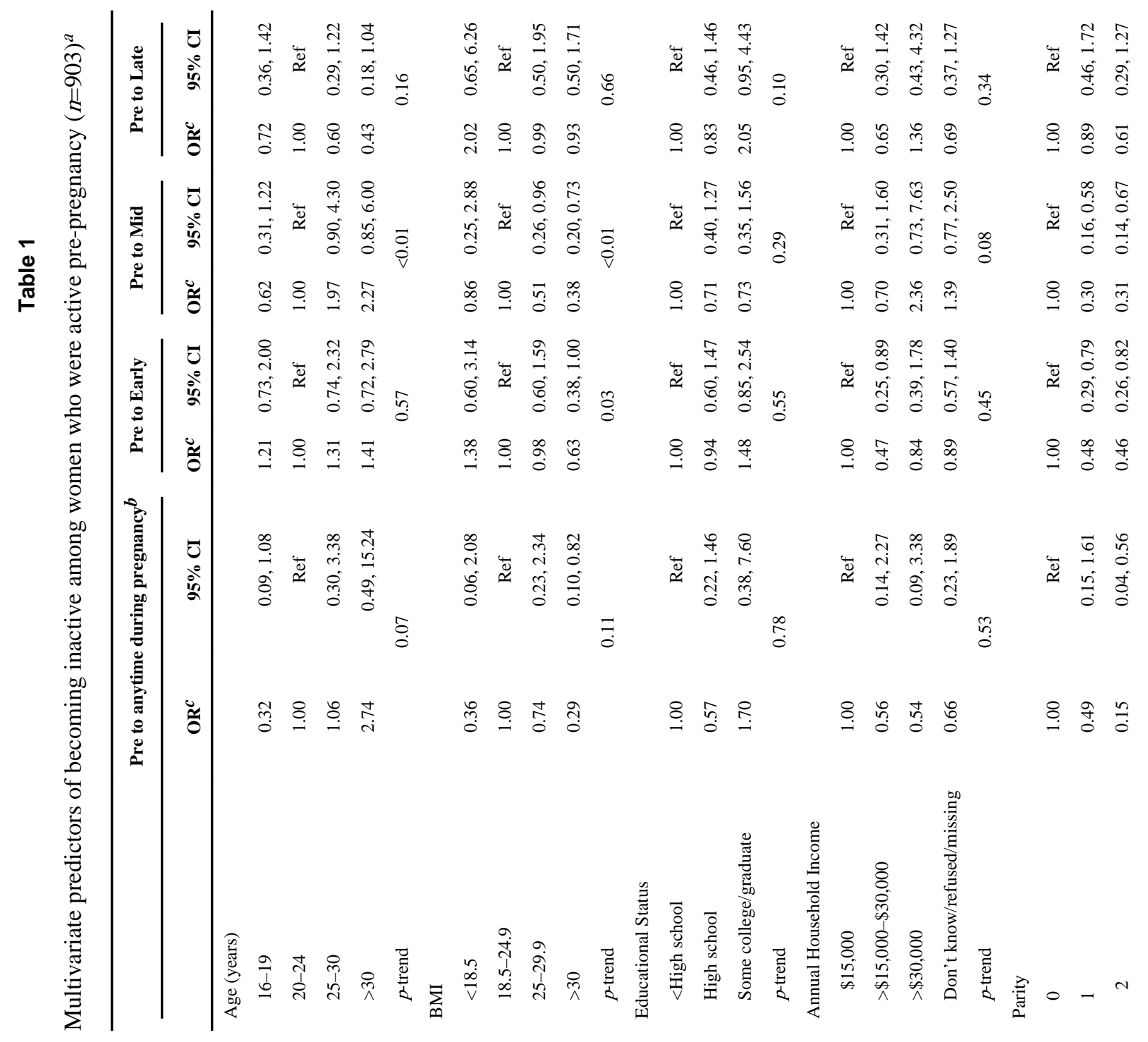

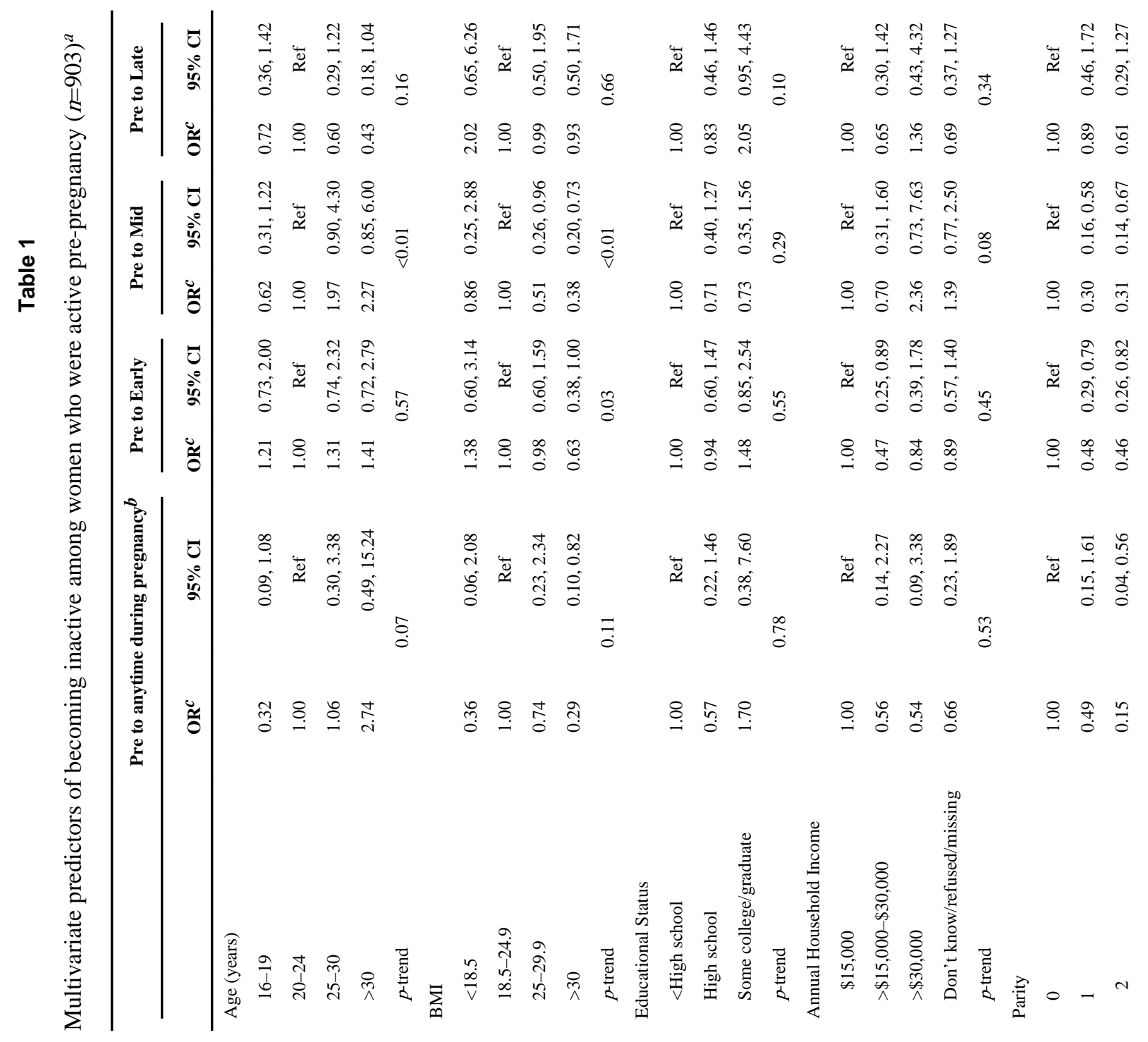

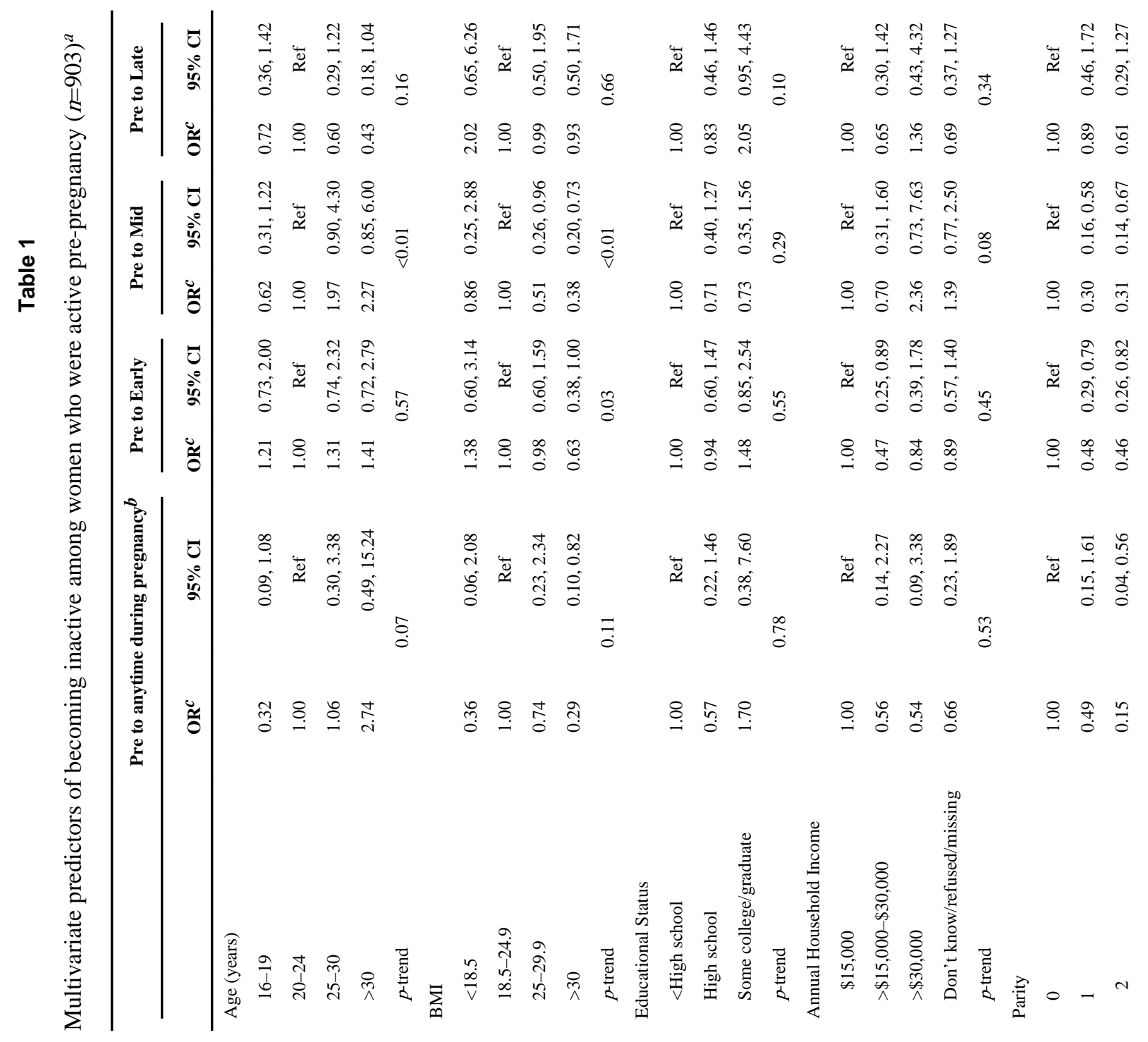

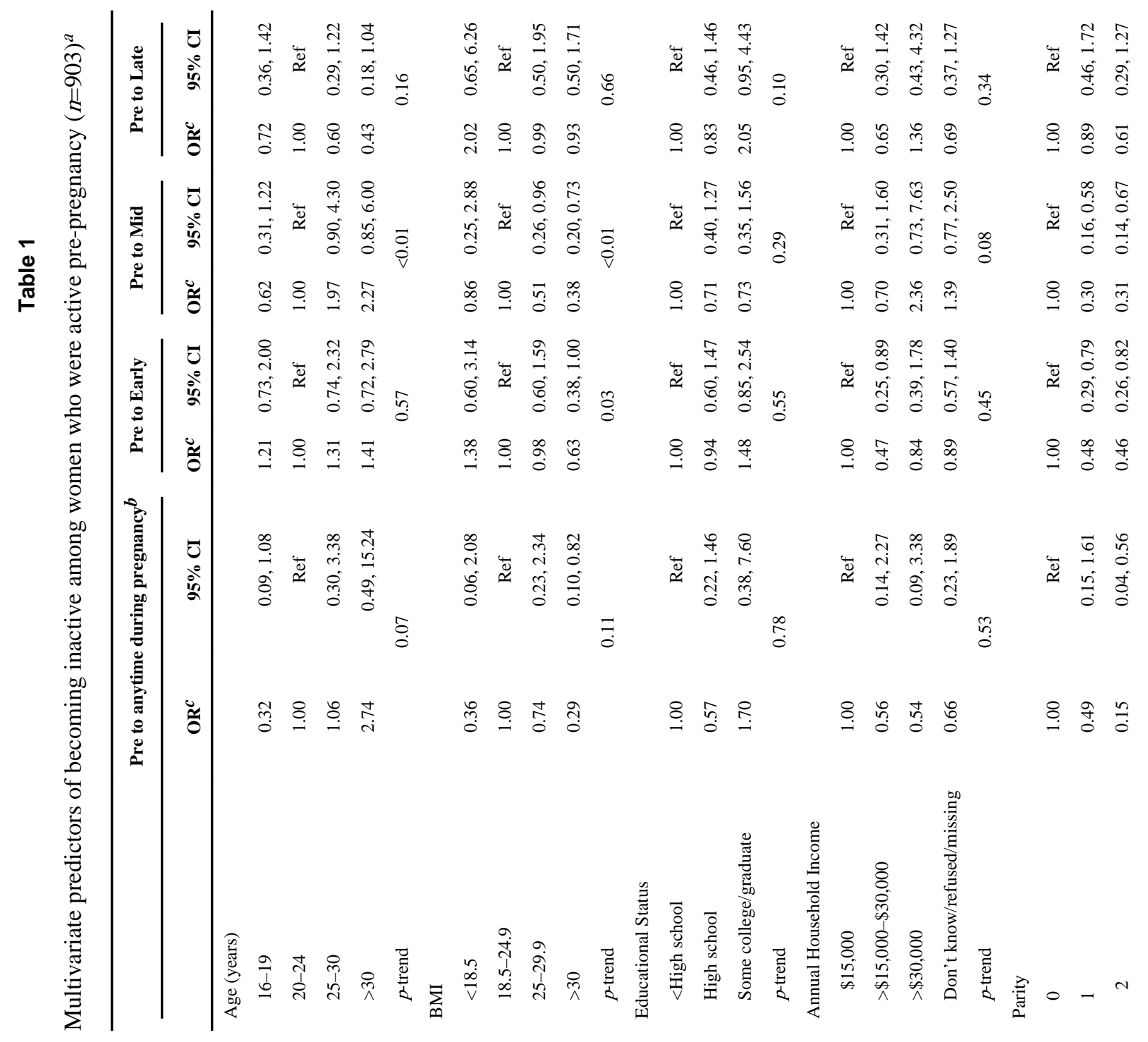




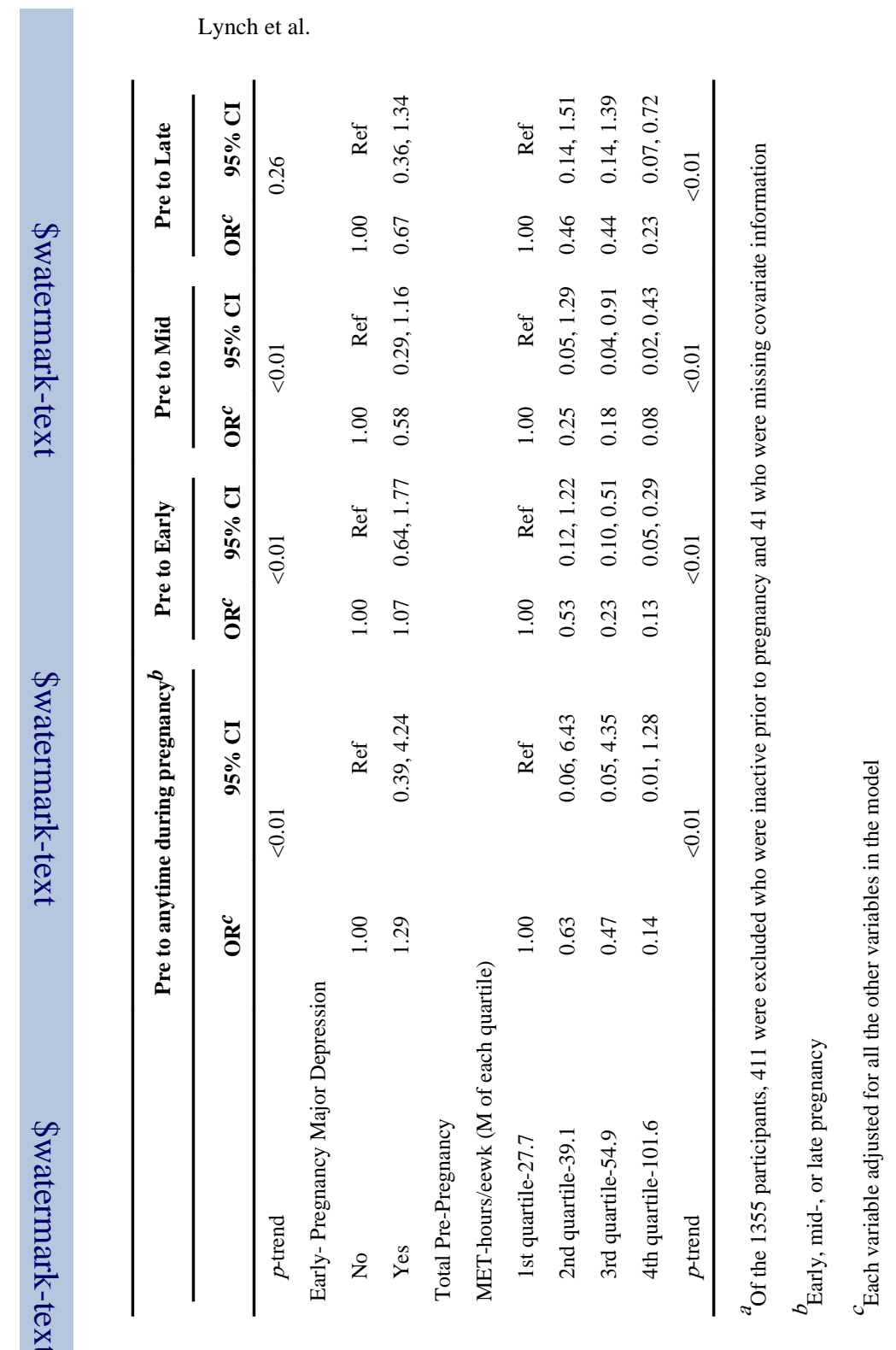

Page 7 


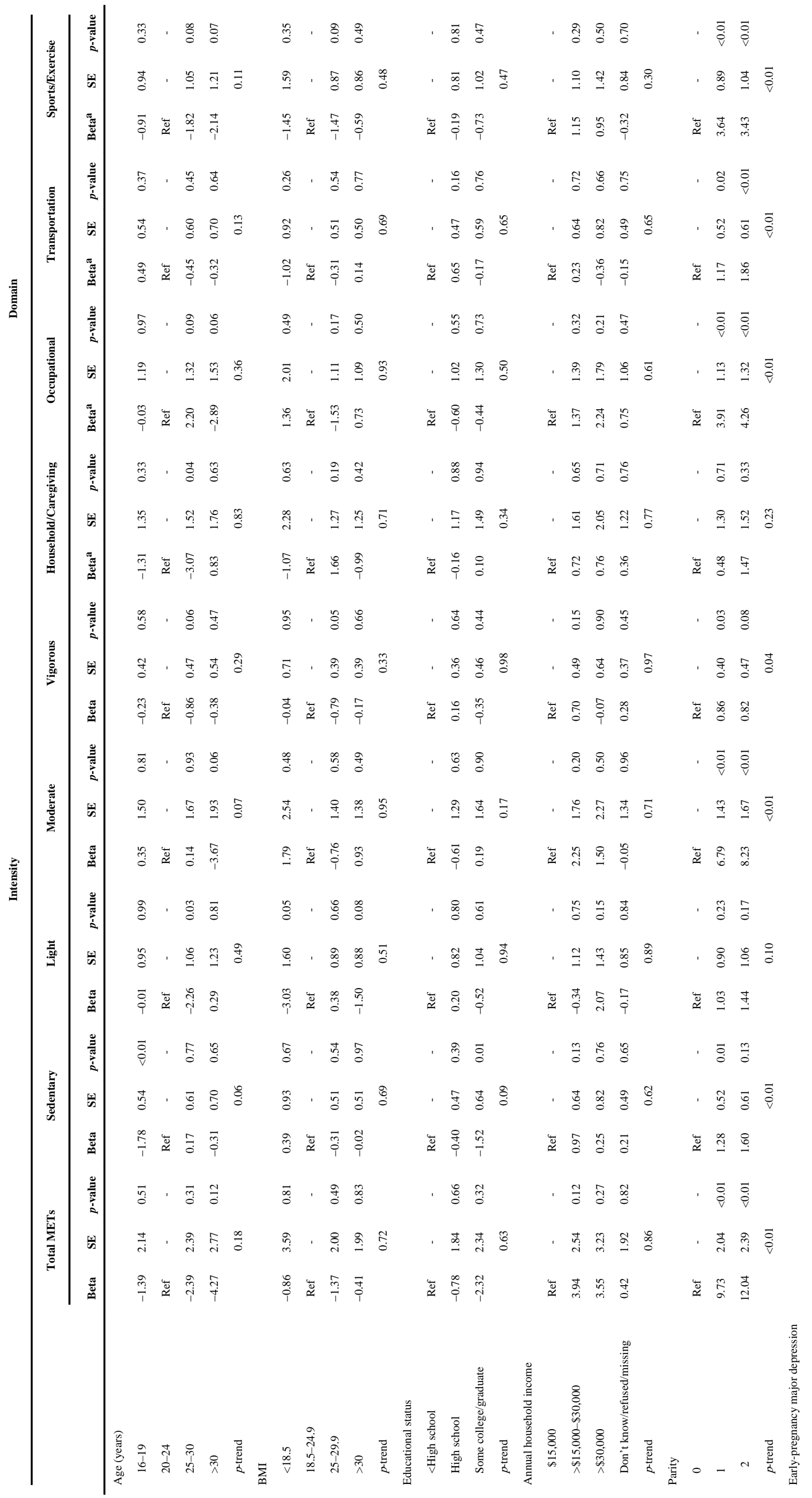




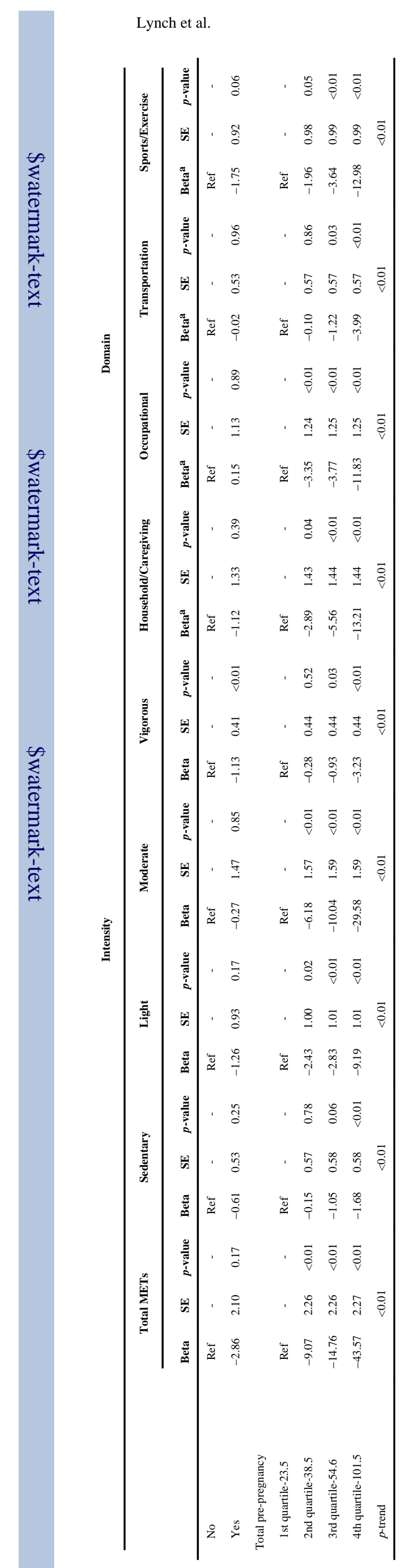

Page 9 